\title{
Redução de hemoglobina: risco para lesão renal aguda após revascularização do miocárdio
}

\author{
Hemoglobin decrease: risk for acute kidney injury after myocardial revascularization \\ Reducción de la hemoglobina: riesgo de lesión renal aguda después de la revascularización miocárdica
}

\author{
Tayse Tâmara da Paixao Duarte', Wellington Luiz de Lima", \\ Alisson Henrique Santos Ribeiro"l', Marcia Cristina da Silva Magro'v
}

\begin{abstract}
RESUMO
Objetivo: verificar se a redução da taxa de hemoglobina no pós-operatório de revascularização do miocárdio interfere na função renal dos pacientes. Método: estudo observacional e prospectivo, desenvolvido entre fevereiro e junho de 2016, com 51 pacientes que realizaram cirurgia de revascularização do miocárdio em um hospital especializado em cardiologia do Distrito Federal. Os dados foram coletados por meio de um instrumento estruturado. 0 teste exato de Fisher, Qui-quadrado e KruskalWallis foram empregados para análise estatística. Considerou-se significativo resultados com $\mathrm{p} \leq 0,05$. Projeto de pesquisa aprovado pelo Comitê de Ética, protocolo 44999215.9.0000.0026. Resultados: entre os pacientes, 45 (78,9\%) evoluíram com lesão renal aguda após revascularização do miocárdio. Idade mais elevada $(64 \pm 9$ anos, $p=0,05)$, tempo de circulação extracorpórea $(p=0,05)$, índice de massa corporal $(p=0,02)$, uso de antibióticos $(p=0,03)$ e redução da taxa de hemoglobina $(p=0,04)$, contribuíram para lesão renal aguda. Conclusão: a redução da taxa de hemoglobina foi associada estatisticamente à lesão renal aguda após revascularização do miocárdio.
\end{abstract}

Descritores: Enfermagem; Revascularização Miocárdica; Hemoglobinas; Lesão Renal Aguda.

\section{ABSTRACT}

Objective: to determine whether decreased hemoglobin rate after myocardial revascularization surgery interferes with patients' renal function. Method: this prospective, observational study was conducted between February and June 2016 with 51 patients who underwent myocardial revascularization surgery at a specialist cardiology hospital specializing in the Federal District. Data were collected using a structured instrument. The Fisher's exact, Chi-square and Kruskal-Wallis tests were used for statistical analysis. Results were considered significant at $p \leq 0.05$. The research project was approved by the research ethics committee (Protocol 44999215.9.0000.0026). Results: it was observed that 45 patients (78.9\%) developed acute kidney injury after myocardial revascularization. Oldest age ( $64 \pm 9$ years, $p=0.05)$, cardiopulmonary bypass time $(p=0.05)$, body mass index $(p=0.02)$, use of antibiotics $(p=0.03)$, and decreased hemoglobin rate $(p=0.04)$ contributed to acute kidney injury. Conclusion: decreased hemoglobin rate was statistically associated with acute kidney injury following myocardial revascularization. Descriptors: Nursing; Myocardial Revascularization; Hemoglobins; Acute Kidney Injury.

\section{RESUMEN}

Objetivo: verificar si la reduciendo la tasa de hemoglobina en el postoperatorio de revascularización miocárdica interfiere con la función renal de los pacientes. Método: estudio observacional prospectivo realizado entre febrero y junio de 2016 con 51 pacientes que se sometieron a una cirugía de revascularización miocárdica en un hospital especializado en cardiología en el Distrito Federal. Los datos fueron recolectados utilizando un instrumento estructurado. La prueba exacta de Fisher, Chicuadrado y Kruskal-Wallis se utilizaron para el análisis estadístico. Los resultados se consideraron significativos con $p \leq 0.05$. Proyecto de investigación aprobado por el Comité de Ética, protocolo 44999215.9.0000.0026. Resultados: se observó que 45 $(78,9 \%)$ pacientes desarrollaron lesión renal aguda después de la revascularización miocárdica. Edad avanzada (64 \pm 9 años, $p$ $=0.05)$, tiempo de circulación corporal extra $(p=0.05)$, índice de masa corporal $(p=0.02)$, uso de antibióticos $(p=0.03)$ y tasa de hemoglobina reducida $(p=0.04)$, contribuyó a lesión renal aguda. Conclusión: reduciendo la tasa de hemoglobina contribuyó a lesión renal aguda después de la revascularización miocárdica.

Descriptores: Enfermería; Revascularización Miocárdica; Hemoglobinas; Lesión Renal Aguda.

\section{INTRODUÇÃO}

Vários fatores associados à cirurgia cardíaca, incluindo o pinçamento da aorta, circulação extracorpórea (CEC), taxa de transfusão sanguínea e dose elevada de vasopressores, podem induzir ciclos de isquemia e reperfusão, aumentar o dano oxidativo e a inflamação renal e sistêmica e contribuir para a instalação da lesão renal aguda (LRA) ${ }^{1}$.

'Enfermeira. Mestre em Ciências da Saúde. Doutoranda pelo Programa de Pós-Graduação em Enfermagem da Universidade de Brasília. Professora Assistente da Faculdade de Ceilândia. Brasília, Brasil. E-mail: taysepaixao@unb.br. ORCID: https://orcid.org/0000-0003-1608-618X

"Enfermeiro. Especialista. Mestrando pelo Programa de Pós-Graduação em Enfermagem da Universidade de Brasília. Brasília, Brasil. E-mail: wellingtonporteiras@hotmail.com. ORCID: https://orcid.org/0000-0002-7023-7244

"'Enfermeiro. Residente de Enfermagem em Cardiologia no Hospital Sírio Libanês. São Paulo, Brasil. E-mail: alisson.henriqe@gmail.com. ORCID: https://orcid.org/0000-0003-4056-3062 IVEnfermeira. Doutora em Enfermagem. Professora Associada da Faculdade de Ceilândia. Universidade de Brasília. Brasília, Brasil. E-mail: marciamagro@unb.br ORCID: https://orcid.org/0000-0002-4566-3217 
A incidência de LRA após cirurgia de revascularização do miocárdio (CRM) tem se mantido elevada e pode acometer cerca de $54 \%$ dos pacientes, contribuindo substancialmente para o aumento da morbimortalidade a curto e longo prazo, o que gera inevitavelmente elevados custos à saúde $\mathrm{e}^{1,2}$

A circulação extracorpórea (CEC), frequente em cirurgias cardíacas, contribui para coagulação sanguínea por disfunção plaquetária, diminuição dos fatores de coagulação e aumento da atividade fibrinolítica ${ }^{3}$. Embora o tempo de CEC seja reconhecido como fator de risco para LRA, a associação entre alteração das taxas de hemoglobina, necessidade de transfusão sanguínea e a disfunção renal ainda não estão bem esclarecidas ${ }^{4,5}$. Acredita-se que a redução de hemoglobina circulante, decorrente da hemólise e o aumento da hemoglobina plasmática livre pela ressuscitação volêmica agressiva sejam condições multifatoriais que podem contribuir para o desenvolvimento da LRA por desencadear hipóxia renal e, elevar o consumo de oxigênio e estresse oxidativo tubular renal ${ }^{6}$.

A maioria dos fatores de risco para LRA após cirurgia cardíaca classifica-se como não modificável, no entanto a anemia, a taxa de hemoglobina e transfusão sanguínea tem sido reconhecidos como potencialmente modificáveis, ainda que não estejam completamente esclarecidos os mecanismos desencadeantes do insulto renal, nesse cenário. A interrelação entre essas variáveis e LRA ainda não têm se mostrado amplamente relatada ${ }^{7}$, o que motivou o desenvolvimento deste estudo.

Desta forma a compreensão dos fatores de risco pode contribuir para planejamento de ações de prevenção da LRA, fundamental no ambiente hospitalar, onde a susceptibilidade de um paciente e a administração de agentes potencialmente nefrotóxicos podem ser avaliados antes da cirurgia. Identificar os pacientes suscetíveis à LRA oferece oportunidade para a equipe de saúde otimizar a gestão do cuidado em situações de alto risco, como alteração da taxa de hemoglobina, e assim melhorar o monitoramento e controle ${ }^{6}$.

Neste contexto, a enfermagem tem papel fundamental e insubstituível em identificar os pacientes com risco potencial para desenvolver LRA, contribuindo para minimizar ou impedir a evolução de uma patologia aguda para uma condição permanente e com graves complicações, por meio da identificação de indicadores de risco (doenças crônicas não transmissíveis, diabetes mellitus, hipertensão arterial, doença renal crônica, pré-existentes e idade avançada), melhorando tanto a gestão como a qualidade assistencial a partir de estratégias de monitoramento laboratorial e hemodinâmico.

Sendo assim, este estudo tem como objetivo verificar se a redução da taxa de hemoglobina no pós-operatório de revascularização do miocárdio interfere na função renal dos pacientes.

\section{MÉTODO}

Trata-se de um estudo observacional, longitudinal e prospectivo, de natureza quantitativa, desenvolvido entre fevereiro e junho de 2016 em uma Unidade de Terapia Intensiva (UTI) geral de um hospital privado especializado em cardiologia do Distrito Federal, Brasil.

Dos 122 pacientes adultos internados na UTI durante o acompanhamento, 57 foram incluídos de forma consecutiva por conveniência. Os motivos das perdas foram tempo de internação, óbito ou transferência, em período de até 48 horas de internação na terapia intensiva. Foram incluídos pacientes com idade igual ou superior a 18 anos, submetidos exclusivamente a cirurgia de revascularização do miocárdio, sem histórico de lesão renal prévia. Foram excluídos pacientes submetidos a exames contrastados nas 48 horas anteriores ao procedimento cirúrgico e com hemoglobina menor que $8 \mathrm{~g} / \mathrm{dL}$.

Para avaliação da função renal adotou-se o critério creatinina da classificação KDIGO (Kidney Disease Improving Global Outcome $)^{8}$. Essa classificação define LRA como o aumento de $0,3 \mathrm{mg} / \mathrm{dL}$ da creatinina basal em tempo menor ou igual a 48 horas, ou ainda redução do débito urinário $<0,5 \mathrm{~mL} / \mathrm{kg} /$ hora por 6 horas. Ela estratifica de forma progressiva a gravidade da LRA em estágios, 1 (risco), 2 (lesão renal) e 3 (falência renal), adotando como referência o pior valor da creatinina sérica ${ }^{9}$. Para fins do estudo adotou-se o último resultado de creatinina sérica dosado anteriormente à internação na UTI, como baseline, mas quando indisponível considerou-se o menor valor identificado nos sete primeiros dias após internação na UTI ${ }^{9}$.

Os dados foram coletados diariamente, desde admissão do paciente na UTI até o momento de alta para unidade de internação, por meio de um instrumento estruturado de coleta, constituído das variáveis: dados de identificação, demográficos, clínicos (doenças pregressas, medicamentos em uso e exames laboratoriais pré-cirurgia), cirúrgicos (tempo de cirurgia, tempo de circulação extracorpórea, uso de drogas vasoativas, tempo de intubação, exames laboratoriais pós-cirurgia e tipo de cirurgia), período de internação na UTI e índice prognóstico Acute Physiology and Chronic Health Evaluation II (APACHE II) ${ }^{10}$. 
Os parâmetros laboratoriais e hemodinâmicos foram extraídos dos registros dos prontuários dos pacientes, sendo eles: pressão sistólica alterada quando $<90$ ou $>140 \mathrm{mmHg}$, pressão diastólica alterada quando $<60 \mathrm{ou}>100 \mathrm{mmHg}$, hematócrito alterado quando $<35 \%$ ou $>47 \%$, hemoglobina alterado quando $<11,7 \mathrm{~g} / \mathrm{dL}$, lactato alterado quando $<20$ $\mathrm{mg} / \mathrm{dl}$, acidose quando $\mathrm{pH}$ arterial $<7,35$ e alcalose quando $\mathrm{pH}$ arterial $>7,45$, leucocitose (elevação no número de leucócitos) quando $>11.000 \mathrm{~mm}^{3}$.

Para análise estatística, os pacientes foram divididos em dois grupos, aqueles que não apresentaram (12 pacientes) e os que apresentaram LRA (45 pacientes), de qualquer gravidade segundo a classificação KDIGO (estágios $1,2$ e 3$)$.

Os resultados foram expressos em estatística descritiva, por meio de frequência absoluta (n), frequência relativa (\%), média e desvio padrão. Os dados categóricos foram analisados pelos testes Chi-quadrado com correção de Yates, MannWhitney e exato de Fisher. Foram considerados significativos resultados com valor de $p \leq 0,05$

Obedecendo a Resolução 466/2012, este projeto foi submetido e aprovado pelo Comitê de Ética em Pesquisa do Instituto de Cardiologia do Distrito Federal sob protocolo 44999215.9.0000.0026.

\section{RESULTADOS}

Foram acompanhados 57 pacientes, havendo predominência do sexo feminino (54,4\%) e idade média de $63 \pm 9$ anos. $O$ índice de massa corporal (IMC) foi de $29,7 \pm 5,23 \mathrm{~kg} / \mathrm{m}^{2}$. Quanto à função cardíaca, 43,9\% apresentaram fração de ejeção menor que $50 \%$, e $10,5 \%$ inferior a $40 \%$. As comorbidades mais frequentes foram hipertensão arterial sistêmica (HAS) $(75,4 \%)$ e dislipidemias (61,4\%). De acordo com as variáveis biológicas, observou-se alteração de hematócrito em todos os pacientes, e de hemoglobina em $96,5 \%$ dos casos. 0 desequilíbrio ácido-base foi identificado em $89,4 \%$ dos pacientes, e alteração do lactado em $71,9 \%$. A maioria dos pacientes $(98,2 \%)$ evoluiu com alta da UTI em $4,9 \pm 8,8$ dias.

Segundo o critério débito urinário da classificação KDIGO, 38,6\% apresentaram LRA de menor gravidade (risco), 17,6\% LRA de gravidade intermediária (lesão renal) e 1,8\% apresentou LRA de maior gravidade (falência renal). Quando à função renal foi avaliada pelo critério creatinina sérica predominou entre os pacientes o estágio de menor gravidade (risco) $(49,1 \%)$, mas ainda 5,3\% dos pacientes foi classificada no intermediário (lesão) e 1,8\% no estágio 3 , de falência renal.

Pacientes com idade avançada evoluíram de forma significativa com LRA ( $p=0,05)$, assim como aqueles com história de alcoolismo $(p=0,04)$. Dentre os $45(78,9 \%)$ pacientes que desenvolveram lesão renal aguda, $36(80 \%)$ tinham diagnóstico médico de hipertensão arterial e $21(46,7 \%)$ de diabetes mellitus, embora não tenha se observado uma correlação estatística significativa entre essas doenças crônicas e o desenvolvimento da LRA (Tabela 1).

Em relação aos aspectos cirúrgicos, o tempo de circulação extracorpórea (CEC) mostrou-se associado ao desenvolvimento de LRA $(p=0,05)$, ao contrário do tempo de anoxia $(p=0,4)$ e do APACHE II $(p=0,5)$. Quase todos os pacientes receberam como enxerto miocárdico a artéria mamária interna esquerda (93\%) e mais da metade necessitou de três enxertos miocárdicos (61,4\%). As cirurgias com CEC predominaram (96,5\%) e o seu tempo de médio foi de 93 minutos com anóxia de 79 minutos.

A principal complicação pós-operatória foi o aumento da contagem de leucócitos (leucocitose, acima de 11.000 $\mathrm{mm}^{3}$ ) em $14 \%$ dos pacientes, seguido da congestão pulmonar (7\%). O uso de diurético foi observado em mais de $50 \%$ dos pacientes (73,7\%). Todos apresentaram alterações de hematócrito (100\%) e a hemoglobina se apresentou reduzida $(<11,7 \mathrm{~g} / \mathrm{dL}$ ) em $96,5 \%$ dos casos, o que justificou a necessidade de transfusão sanguínea em $54,4 \%$ dos casos. A maioria dos pacientes evoluiu com LRA (78,9\%). Observou-se que todos os pacientes com lesão renal apresentaram alteração de hemoglobina sérica e $57 \%$ necessitaram de transfusão sanguínea, sendo a relação entre alteração da taxa de hemoglobina e LRA significativa pelo teste de Fisher $(p=0,04)$.

No que tange às medicações utilizadas, o uso de antibióticos para o tratamento de infecções relacionadas à assistência em saúde, mostrou associação significativa para o desenvolvimento de falência renal $(p=0,03)$. 0 tempo médio de ventilação mecânica (VM) foi de $840 \pm 320$ minutos. Pacientes que desenvolveram LRA necessitaram de maior tempo de VM, embora essa relação não tenha sido significativa $(p=0,1)$. Todos os pacientes fizeram uso de drogas vasoativas (DVA), a exemplo da noradrenalina (84,2\%), mas como inotrópico positivo a dobutamina se destacou (96,5\%).

Os doentes que evoluíram nos estágios de lesão e falência renal (estágios 2 e 3, KDIGO) de comprometimento da função renal $(n=45)$ também evidenciaram alterações de hemoglobina em $100 \%$ dos casos $(p=0,04)$, desses a maioria necessitou de transfusão sanguínea $(66,7 \%)(p=0,3)$. 
TABELA 1: Correlação da lesão renal aguda com as características clínicas de pacientes em pós-operatório de revascularização do miocárdio $(n=57)$. Distrito Federal, Brasil, 2016

\begin{tabular}{|c|c|c|c|}
\hline Características & $\begin{array}{c}\text { Sem LRA } \\
(n=12)\end{array}$ & $\begin{array}{c}\text { Com LRA } \\
(n=45)\end{array}$ & Valor p \\
\hline Sexo feminino & $6(50,0)$ & $25(55,6)$ & 0,7 \\
\hline Idade (anos) ${ }^{a}$ & $59 \pm 8$ & $64 \pm 9$ & 0,05 \\
\hline IMC* (kg/m2)a,ii & $26,8 \pm 4,3$ & $27,2 \pm 4,3$ & 0,7 \\
\hline \multicolumn{4}{|l|}{ Comorbidades } \\
\hline Diabetes & $3(25,0)$ & $21(46,7)$ & 0,2 \\
\hline Hipertensão & $7(58,3)$ & $36(80,0)$ & 0,1 \\
\hline Alcoolismo & $2(16,7)$ & $0(0,0)$ & 0,04 \\
\hline Tabagismo & $4(33,3)$ & $15(33,3)$ & 0,6 \\
\hline DLP & $5(41,7)$ & $30(66,7)$ & 0,1 \\
\hline \multicolumn{4}{|l|}{ Desfecho Hospitalari } \\
\hline Alta & $12(100,0)$ & $42(93,3)$ & \multirow{2}{*}{0,8} \\
\hline Óbito & $0(0,0)$ & $1(2,2)$ & \\
\hline \multicolumn{4}{|l|}{ Desfecho UTI } \\
\hline Alta & $12(100,0)$ & $44(97,8)$ & \multirow{2}{*}{0,8} \\
\hline Óbito & $0(0,0)$ & $1(2,2)$ & \\
\hline Pressão Sistólica alterada & $7(58,3)$ & $38(84,4)$ & 0,06 \\
\hline Pressão Diastólica alterada & $9(75,0)$ & $42(93,3)$ & 0,1 \\
\hline Hemoglobina $<11,7 \mathrm{~g} / \mathrm{dL}$ & $10(83,3)$ & $45(100,0)$ & 0,04 \\
\hline Lactato alterado & $10(83,3)$ & $31(68,9)$ & 0,3 \\
\hline Alcalose & $3(25,0)$ & $5(11,1)$ & 0,2 \\
\hline Acidose & $7(58,3)$ & $36(80,0)$ & 0,1 \\
\hline \multicolumn{4}{|c|}{$\begin{array}{l}\text { a média } \pm \text { DP; '2 pacientes sem informação, "i } 1 \text { paciente sem informação, iii } 4 \\
\text { pacientes sem informação; Teste Chi-quadrado com correção de Yates, } \\
\text { Exato de Fisher, Teste de Mann-Whitney; Pressão Sistólica alterada quando } \\
<90 \text { ou > } 140 \mathrm{mmHg} \text {; Pressão Diastólica alterada quando }<60 \text { ou }>100 \\
\mathrm{mmHg} \text {; Lactato alterado quando }<20 \mathrm{mg} / \mathrm{dl} \text {; Acidose quando } \mathrm{PH} \text { arterial < } \\
\text { 7,35; Alcalose quando } \mathrm{PH} \text { arterial }>7,45 \text {. }\end{array}$} \\
\hline
\end{tabular}

\section{DISCUSSÃo}

A redução da taxa de hemoglobina embora não seja reconhecida como fator exclusivo para LRA $^{11}$, os nossos resultados mostraram que a sua redução possui efeito indutor da LRA desencadeando a necessidade de transfusão sanguínea no pós-operatório de revascularização do miocárdio. Evidências científicas têm apontado uma associação direta entre a queda de hemoglobina pós-operatória e a consequente necessidade de transfusão sanguínea e o aumento da taxa de LRA ${ }^{4,11}$.

Os mecanismos relacionados ao desenvolvimento de LRA no pós-operatório de cirurgia cardíaca originam-se da redução de perfusão renal desencadeada pela circulação extracorpórea ${ }^{7}$. A redução de hemoglobina, como identificado no presente estudo se manifesta como vilã interferindo no adequado funcionamento do sistema renal ao aumentar o risco do paciente evoluir com LRA ${ }^{12}$.

A queda na taxa de hemoglobina ao desencadear necessidade de transfusão, gera uma resposta pró-inflamatória e assim, prejudica a distribuição de oxigênio ao parênquima renal exarcebando o estresse oxidativo dos tecidos. Isto por sua vez, em pacientes com associação de comorbidades pode aumentar a predisposição à LRA ${ }^{13}$.

No contexto geral, a incidência de LRA mostrou-se elevada $(78,9 \%)$ e quando comparada a estudos prévios ${ }^{4,14}$ mostrou superioridade em relação aos valores encontrados $(8,29 \%$ e $37,5 \%)$ em outros estudos, a partir do emprego do mesmo critério de classificação, KDIGO, durante o período pós-operatório de cirurgia cardíaca. A classificação KDIGO tem sido implementada em práticas clínicas e cirúrgicas para subsidiar a detecção precoce e o delineamento de medidas adicionais para fins de controle da progressão do dano renal. Ela possibilita uma avaliação padronizada da gravidade da LRA e estimativas para as medidas epidemiológicas ${ }^{15}$.

No período de pós-operatório de cirurgia cardíaca os percentuais elevados de LRA em cuidados críticos geralmente são decorrentes de diversos fatores como idade, uso de CEC prolongada, uso de drogas nefrotóxicas, IMC elevado, alteração das taxas de hemoglobina e transfusão sanguínea ${ }^{16,17}$. Condições também identificadas no presente estudo. 
A idade avançada também é reconhecida como fator de risco para LRA ${ }^{4,18}$, dada redução fisiológica do fluxo sanguíneo renal e a menor capacidade de adaptação hemodinâmica desses pacientes, quando expostos a CEC e drogas nefrotóxicas ${ }^{19}$. O tempo prolongado de CEC tem se mostrado como uma das principais causas de LRA no pós-operatório de cirúrgica cardíaca ${ }^{20}$, além de predispor a redução de hemoglobina e deflagração da necessidade de transfusão de hemocomponentes, exacerbando risco de comprometimento renal ${ }^{21}$.

Dentre as drogas nefrotóxicas se destacam as drogas vasoativas e os antibióticos ${ }^{22}$. Estes representam uma das principais classes de medicamentos causadoras de danos aos túbulos renais e, portanto, indutores de LRA. Causam, ainda, alterações hemodinâmicas, nefrite intersticial aguda, além de proteinúria nefrótica, aumentando a suscetibilidade à lesão glomerular ${ }^{23}$.

No panorama geral, o uso de álcool se associou ao desenvolvimento de LRA. Evidência científica, assim como neste estudo, mostrou que o uso prolongado do álcool pode desencadear lesões celulares em diversos tecidos do corpo, inclusive nas células renais e ainda determinar necrose tecidual e desenvolvimento de LRA $^{24}$. Embora não tenha se associado de forma significativa para LRA, a acidose metabólica esteve presente em $80 \%$ daqueles que apresentaram lesão renal. A acidose metabólica é considerada um fator nefrotóxico associado à progressão da doença renal. Acreditase que o mecanismo envolva a geração intersticial aumentada de amônia (com a ativação da via do complemento), produção local aumentada de endotelina e a ativação do sistema renina-angiotensina- aldosterona (SRAA). Estes fatores predispõem a fibrose renal intersticial e a aceleração da perda de massa muscular, além de determinar elevações da morbimortalidade nos pacientes com lesão renal ${ }^{25}$.

Neste contexto, destaca-se a importância da assistência de enfermagem no ambiente de terapia intensiva, onde cuidados cirúrgicos imediatos centrados nos pacientes são necessários para manutenção da segurança. O trabalho do enfermeiro na equipe multidisciplinar constitui-se em um pilar, frente aos desafios tecnológicos que tangenciam a complexidade do cuidado em saúde e deve fundamentar-se por evidências científicas ${ }^{26}$.

Constata-se, nessa perspectiva, que ao assumir a importância do cuidado centrado no paciente, compete ao enfermeiro, em meio as responsabilidades no processo de gestão do cuidado, a realização de exame físico detalhado, execução de procedimentos e intervenções relativas ao tratamento, além de avaliação de exames laboratoriais, à exemplo da hemoglobina sérica. Reconhece-se que o enfermeiro enquanto responsável pela gestão do cuidado ao paciente deve atuar de forma direcionada ao alcance da qualidade dos serviços de saúde com ênfase no cuidado integral centrado às necessidades humanas ${ }^{26}$.

\section{CONCLUSÃO}

Pacientes submetidos a revascularização do miocárdio com redução da taxa de hemoglobina evoluíram significativamente com LRA. Fatores como idade, o tempo de CEC, o uso de antibióticos e o alcoolismo contribuíram ainda para a LRA.

Este estudo reforça a repercussão da queda da taxa de hemoglobina na função renal no cenário de pós-operatório. Limitações no controle de hemoglobina sérica e na avaliação criteriosa da necessidade de transfusão sanguínea se destacam entre as condições clínicas que podem predispor ao comprometimento renal.

Conhecer os fatores relacionados à ocorrência de LRA contribui para direcionamento de profissionais de saúde, inclusive enfermeiros, possibilitando o desenho de uma avaliação sistematizada em saúde, assim como planos de intervenção individualizados favorecendo a prevenção do dano renal e impedindo a progressão do evento agudo para crônico. As limitações deste estudo se concentram em um pequeno tamanho amostral e realização em um único centro.

Espera-se que este estudo possa subsidiar a prática de saúde, pautada na abordagem interdisciplinar, caracterizada pela interação da equipe de enfermagem com as demais equipes de saúde para reconhecimento de fatores de risco relacionados a lesão renal aguda e ainda contribua à promoção de intervenções individualizadas na gestão do cuidado.

\section{REFERÊNCIAS}

1. Kang W, Wu X. Pre-, intra-, and post-operative factors for kidney injury of patients underwent cardiac surgery: a retrospective cohort study. Med Sci Monit [Internet], 2019 [cited 2020 Mar 20]; 25:5841-49. DOI: http://dx.doi.org/10.12659/MSM.915996

2. Kertai MD, Zhou S, Karhausen JA, Cooter M, Jooste E, Li YJ, et al. Platelet counts, acute kidney injury, and mortality after coronary artery bypass grafting surgery. Anesthesiology [Internet], 2016 [cited 2020 Mar 20]; 124(2):339-52. DOI: http://dx.doi.org/10.1097/ALN.0000000000000959

3. Tobey R, Cheng H, Gao M, Li Z, Young JN, Boyd WD, et al. Postoperative acute kidney injury and blood product transfusion after synthetic colloid use during cardiac surgery. J Cardiothorac Vasc Anesth [Internet], 2017 [cited 2020 Mar 20]; 31(3):853-62. DOI: http://dx.doi.org/ 10.1053/j.jvca.2016.12.024 
4. Freeland K, Hamidian AJ, Duvall LM, Mancini MC. Postoperative blood transfusion is an independent predictor of acute kidney injury in cardiac surgery patients. J Nephropathol [Internet], 2015 [cited 2020 Mar 20]; 4(4):121-6. DOI: http://dx.doi.org/10.12860/jnp.2015.23

5. Garg AX, Shehata N, McGuinness S, Whitlock R, Fergusson D, Wald R, et al. Risk of acute kidney injury in patients randomized to a restrictive versus liberal approach to red blood cell transfusion in cardiac surgery: a substudy protocol of the transfusion requirements in cardiac surgery III noninferiority Trial. Can J Kidney Health Dis [Internet], 2018 [cited 2020 Mar 20]; 5:1-8. DOI: http://dx.doi.org/10.1177/2054358117749532

6. O'Neal JB, Shaw AD, Billings FT. Acute kidney injury following cardiac surgery: current understanding and future directions. Crit Care [Internet], 2016 [cited 2020 Mar 22]; 20:187. DOI: http://dx.doi.org/10.1186/s13054-016-1352-z

7. Karkouti K, Grocott HP, Hall R, Jessen ME, Kruger C, Lerner AB, et al. Interrelationship of preoperative anemia, intraoperative anemia, and red blood cell transfusion as potentially modifiable risk factors for acute kidney injury in cardiac surgery: a historical multicentre cohort study. Can J Anaesth [Internet], 2015 [cited 2020 Mar 22]; 62(4):377-84. DOI: http://dx.doi.org/10.1007/s12630-014-0302-y

8. Kidney Disease: Improving Global Outcomes (KDIGO). Clinical Practice Guideline for Acute Kidney Injury. Kidney [Internet], 2017 [cited 2020 Mar 22]; 2(1): 1-138. Available from: https://kdigo.org/wp-content/uploads/2016/10/KDIGO-2012-AKI-GuidelineEnglish.pdf

9. Macedo E, Bouchard J, Mehta RL. Renal recovery following acute kidney injury. Curr Opin Crit Care [Internet], 2008 [cited 2020 Mar 22]; 14(6):660-5. DOI: https://dx.doi.org/10.1097/MCC.0b013e328317ee6e

10. Moreno RP, Nassar Júnior AP. Is APACHE II a useful tool for clinical research? Rev Bras Ter Intensiva [Internet], 2017 [cited 2020 Mar 22]; 29(3):264-267. Available from: https://www.scielo.br/pdf/rbti/v29n3/en_0103-507X-rbti-29-03-0264.pdf

11. Gorla R, Tsagakis K, Horacek M, Mahabadi AA, Kahlert P, Jakob H, et al. Impact of preoperative anemia and postoperative hemoglobin drop on the incidence of acute kidney injury and in-hospital mortality in patients with type $b$ acute aortic syndromes undergoing thoracic endovascular aortic repair. Vasc Endovascular Surg [Internet], 2017 [cited 2020 Mar 22]; 51(3):131-8. DOI: http://dx.doi.org/10.1177/1538574417697211

12. Kim-Campbell N, Gretchen C, Callaway C, Felmet K, Kochanek PM, Maul T, et al. Cell-Free plasma hemoglobin and male gender are risk factors for acute kidney injury in low risk children undergoing cardiopulmonary bypass. Crit Care Med [Internet], 2017 [cited 2020 Mar 25]; 45(11):e1123-e1130. Available from: https://www.ncbi.nlm.nih.gov/pmc/articles/PMC5657595/pdf/nihms897500.pdf

13. Duque-Sosa P, Martínez-Urbistondo D, Echarri G, Callejas R, Iribarren MJ, Rábago G, et al. Perioperative hemoglobin area under the curve is an independent predictor of renal failure after cardiac surgery. Results from a Spanish multicenter retrospective cohort study. Plos One [Internet], 2017 [cited 2020 Mar 25]; 12(2):1-14. Available from: https://www.ncbi.nlm.nih.gov/pmc/articles/PMC5321429/pdf/pone.0172021.pdf

14. Li Y, Xu J, Wang Y, Zhang Y, Jiang W, Shen B, et al. A novel machine learning algorithm, Bayesian networks model, to predict the high-risk patients with cardiac surgery-associated acute kidney injury. Clin Cardiol [Internet], 2020 [cited 2020 Mar 25]; 1-10. Available from: https://www.ncbi.nlm.nih.gov/pmc/articles/PMC7368305/pdf/CLC-43-752.pdf

15. Pakula AM, Skinner RA. Acute kidney injury in the critically ill patient: a current review of the literature. J Intensive Care Med [Internet], 2015 [cited 2020 Mar 25]; 31(5):319-24. DOI: http://dx.doi.org/10.1177/0885066615575699

16. Wetz AJ, Richardt EM, Schotola H, Bauer M, Brauer A. Haptoglobin and free haemoglobin during cardiac surgery-is there a link to acute kidney injury? Anaesth Intensive Care [Internet], 2017 [cited 2020 Mar 25]; 45(1):58-66. Available from: https://journals.sagepub.com/doi/pdf/10.1177/0310057X1704500109

17. Joannidis M, Druml W, Forni LG, Groeneveld ABJ, Honore PM, Hoste E, et al. Prevention of acute kidney injury and protection of renal function in the intensive care unit: update 2017: Expert opinion of the Working Group on Prevention, AKI section, European Society of Intensive Care Medicine. Intensive Care Med [Internet], 2017 [cited 2020 Mar 28]; 43(6):730-49. Available from: https://www.ncbi.nlm.nih.gov/pmc/articles/PMC5487598/pdf/134_2017_Article_4832.pdf

18. Santo LS, Romano G, Mango E, lorio F, Savarese L, Numis F, et al. Age and blood transfusion: relationship and prognostic implications in cardiac surgery. J Thorac Dis [Internet], 2017 [cited 2020 Mar 28]; 9(10):3719-27. Available from: https://www.ncbi.nlm.nih.gov/pmc/articles/PMC5723788/pdf/jtd-09-10-3719.pdf

19. Obi AT, Park YJ, Bove P, Cuff R, Kazmers A, Gurm HS, et al. The association ofperioperative transfusion with 30-day morbidityand mortality in patients undergoing majorzvascular surgery. J Vasc Surg [Internet], 2015 [cited 2020 Mar 28]; 61(4):1000-9.e1. DOI: http://dx.doi.org/10.1016/j.jvs.2014.10.106

20. Vives M, Hernandez A, Parramon F, Estanyol N, Pardina B, Muñoz A, et al. Acute kidney injury after cardiac surgery: prevalence, impact and management challenges. Int J Nephrol Renovasc Dis [Internet], 2019 [cited 2020 Mar 28]; 12:153-166. Available from: https://www.ncbi.nlm.nih.gov/pmc/articles/PMC6612286/pdf/ijnrd-12-153.pdf

21. Miranda M, Branco JNR, Vargas GF, Hossne Jr. NA, Yoshimoto MC, Fonseca JH, et al. Analysis of the Use of Extracorporeal Circulation on the In-Hospital Outcomes of Dialytic Patients Who Underwent Myocardial Revascularization Surgery. Arq Bras Cardiol [Internet], 2016 [cited 2020 Mar 28]; 107(6):518-22. Available from: https://www.scielo.br/pdf/abc/v107n6/0066-782Xabc-107-06-0518.pdf

22. Poston JT, Koyner JL. Sepsis associated acute kidney injury. BMJ [Internet], 2019 [cited 2020 Mar 30]; 364:k4891. DOI: https://doi.org/10.1136/bmj.k4891

23. Mehta RL, Awdishu L, Davenport A, Murray PT, Macedo E, Cerda J, et al. Phenotype Standardization for Drug Induced Kidney Disease. Kidney Int [Internet], 2015 [cited 2020 Mar 30]; 88(2):226-34. Available from: https://www.ncbi.nIm.nih.gov/pmc/articles/PMC4758130/pdf/emss-62528.pdf 
24. Bundy JD, Bazzano LA, Xie D, Cohan J, Dolata J, Fink JC, et al. Self-reported tobacco, alcohol, and illicit drug use and progression of chronic kidney disease. Clin J Am Soc Nephrol [Internet], 2018 [cited 2020 Mar 30]; 13(7):993-1001. DOI: https://dx.doi.org/10.2215\%2FCJN.11121017

25. Rezende LR, Souza PB, Pereira GRM, Lugon JR. Metabolic acidosis in hemodialysis patients: a review. J Bras Nefrol [Internet], 2017 [cited 2020 Mar 30]; 39(3):305-11. Available from: https://www.scielo.br/pdf/jbn/v39n3/0101-2800-jbn-39-03-0305.pdf

26. Pereira MCC, Castro SFF, Brito ES , Carvalho NV , Lopes DV , Pinheiro JDS, et al. Nurse's knowledge and practices in the intensive care unit. Rev enferm UFPE on line, 2019 [cited 2020 Mar 30]; 13(1):70-8. Available from:

https://periodicos.ufpe.br/revistas/revistaenfermagem/article/view/234842/31123 